Josef Steinbach, Andrea Holzhauser und Klaus Neudecker

\title{
Die „historische Sozialraumanalyse“ als Instrument zur Identifikation von Planungsproblemen
}

\author{
"Historical Social Area Analysis" \\ as an Instrument for the Identification \\ of Planning Problems
}

\begin{abstract}
Kurzfassung
Zur Erfassung von Problemlagen der Stadtentwicklung kommt die Methode der Sozialraumanalyse seit den 70er Jahren zur Anwendung, allerdings fast nur in Form von Querschnittsanalysen. Hier wird eine umfassende (1971 bis 1991) Längsschnittanalyse für das Wiener Stadtgebiet vorgestellt und mithilfe von dreidimensionalen Trendflächenmodellen visualisiert. Es zeigt sich vor allem, dass die Stadtplanung auf die drohende „doppelte Segregation“ benachteiligter sozialer Gruppen sowie auf den Eintritt der Bevölkerung in neue Alterungszyklen reagieren muss.
\end{abstract}

\section{Abstract}

The method of Social Area Analysis is often used to define problems of urban development, but usually it is based on cross-sectional data only. Here time series (1971-1991) of the input variables are used in order to examine the changes of the Viennese social structure. The results are visualised as three-dimensional trend surfaces. It can be shown that urban planning has to react to "double segregation"-processes of the population as well as to new demographic "cycles of ageing".

\section{Einfuihrung}

In der interdisziplinären Stadtforschung hat die Methode der Sozialraumanalyse eine lange Tradition. Allerdings bleiben die meisten dieser Studien als "Querschnittanalysen“ auf nur einen Beobachtungszeitpunkt beschränkt, zum Teil wegen der fehlenden „historischen“ Daten für längere Perioden der Stadtentwicklung, aber auch wegen verschiedener methodischer Probleme, für die R. J. Horvath (1994) eine elegante und verblüffend einfache Lösung gefunden hat. Sein Verfahren wurde von J. Steinbach und S. Hilger (1997) im Rahmen des Forschungsprojektes „Erneuerungsstrategien für ausgewählte Geschäftszentren im dicht bebauten Wiener Stadtgebiet" angewendet, und zwar zur Erfassung des sozialen Wandels in den Kundeneinzugsbereichen der untersuchten Geschäftsstraßen. Dies gab den Anlass zu einer umfassenden Längsschnittanalyse für das Wiener Stadtgebiet unter

Verwendung der Datenbestände der Volkszählungen bzw. der Häuser- und Wohnungszählungen des Österreichischen Statistischen Zentralamtes für die Jahre 1971, 1981 und 1991. Die Ergebnisse dieser Untersuchung werden hier vorgestellt.

Der erste Abschnitt des vorliegenden Beitrags enthält zunächst einen Überblick über die verwendeten Methoden. Darauf folgt eine kurze Darstellung der Verbreitungsmuster und des räumlich-zeitlichen Wandels der sozioökonomischen, demographischen und ethnischen Strukturen. Abschließend wird auf die Relevanz der Ergebnisse für die Stadtplanung eingegangen, wobei die drohende "doppelte Segregation“ benachteiligter sozialer Gruppen sowie der Eintritt der Bevölkerung in neue Alterungszyklen die wesentlichen Problemlagen bilden. 


\section{Abbildung sozialräumlicher Strukturen}

In der amerikanischen Stadtforschung, wo die sozialen, ökonomischen, demographischen und ethnischen Strukturen größerer Agglomerationen erstmals auf der Basis kleinräumiger Beobachtungseinheiten und unter Verwendung umfangreicher statistischer Datenbestände analysiert wurden, kam es seit den 50er Jahren zur Entdeckung der drei Grunddimensionen der Sozialraumanalyse. Es handelt sich um charakteristische Bündel von Merkmalen, die innerhalb des Stadtgebietes z.T. auch ähnliche räumliche Verbreitungsmuster haben und offenbar einem mehr oder minder allgemein gültigen Ordnungsprinzip entsprechen:

- Die Dimension des "sozioökonomischen Status" wird vor allem von den Indikatoren der abgeschlossenen Schulbildung, des Berufs, des Einkommens, der Wohnverhältnisse und des Mietniveaus gebildet.

- Der "demographische Status" bestimmt sich aus den Kennzahlen des Altersaufbaus, der Größe und der Zusammensetzung der Familien, der Erwerbstätigkeit der Frauen u.a.

- Schließlich kennzeichnet der „ethnische Status“ die räumliche Isolierung von nationalen, ethnischen und rassischen Minderheiten.

E. Shevky und W. Bell (1955, die Begründer der sog. "Sozialraumanalyse") haben diese Grundmuster mit Hilfe von zweidimensionalen Matrizenanalysen von U.S. Census-Daten erstmals erfasst. B.J.L. Berry und Mitarbeitern (1972, die Begründer der sog. „Faktorialökologie") gelang ihre Präzisierung durch den Einsatz des multivariaten statistischen Verfahrens der Faktorenanalyse.

Das Vorherrschen von faktorialökologischen Querschnittsanalysen (für Wien wurde z.B. die erste solche Untersuchung von K. Cserjan und M. Sauberer 1972 durchgeführt) ist natürlich in der oft beschränkten Verfügbarkeit von Daten für mehrere Beobachtungszeitpunkte begründet, aber auch in den Eigenheiten der Faktorenanalyse selbst. Bekanntlich sind ihre Ergebnisse:

- Faktoren als die neuen komplexen Dimensionen oder Indikatoren, die Informationen verdichten (d.h. es werden Inputvariablen mit ähnlichen räumlichen Verbreitungsmustern zusammengefasst) und neue statistische Konstrukte bilden. Mit ihrer Hilfe können bestehende theoretische Konzepte überprüft werden, sie sind aber auch dazu geeignet, um als Ansatzpunkte für die Bildung von neuen Hypothesen zu dienen (K. Überla, 1977).

- Faktorenladungen als Kennwerte, welche den Zusammenhang zwischen den ursprünglichen Variablen und den neuen komplexen Indikatoren beschreiben.

- Faktorenwerte, welche die Ausprägung der neuen Dimensionen für die in der Untersuchung behandelten (räumlichen) Beobachtungseinheiten bemessen.

- Die Faktorenwerte werden auf der Grundlage der sog. Factor Score Coefficients ermittelt, das sind multiple Regressionskoeffizienten, welche den $\mathrm{Zu}-$ sammenhang zwischen den räumlichen Ausprägungsmustern der Faktoren und den Ausgangsdaten erfassen.

Faktorenladungen und Factor Score Coefficients repräsentieren gleichsam die Konstruktionsprinzipien der neuen komplexen Indikatoren. Sie differieren zwischen den verschiedenen Analysen, und zwar immer auch bei sehr ähnlichen Strukturen der Inputvariablen - wie dies meistens der Fall ist, wenn für eine Region getrennte Faktorenanalysen für verschiedene Beobachtungszeitpunkte durchgeführt werden. Bei einem Vergleich der in getrennten Verfahren errechneten räumlichen Ausprägungsmuster der drei Dimensionen der Faktorialökologie gehen die (zeitlichen) Veränderungen der Ergebnisse immer auf zwei Ursachen zurück, deren Einflüsse sich überlagern und nicht zu trennen sind:

- die tatsächliche Variation der Merkmalswerte zwischen den Beobachtungszeitpunkten und

- die Veränderungen in den Konstruktionsprinzipien der Rechenmodelle (Faktorenladungen und Factor Score Coefficients).

Daher ist eine solche Vorgangsweise methodisch nicht korrekt und führt zu verfälschten Ergebnissen (siehe die Diskussion in J. Steinbach, A. Holzhauser, K. Neudecker, 2000). Ein methodisch richtiges Verhalten wurde von R. J. Horvath (1994) angewendet: Er fasste die Datensätze für drei Beobachtungszeitpunkte in einer einzigen Faktorenanalyse zusammen, in der jede Beobachtungseinheit dreimal vertreten ist, also - im Sinne des Konzeptes von T. Hägerstrand - gleichsam drei „Individuen“ im Kontinuum von Raum und Zeit repräsentiert. Somit bezieht sich die auf diesem Weg berechnete Matrix der Faktorenladungen auf die "mittleren" Zusammenhänge zwischen den Inputvariablen und den komplexen Dimensionen über den 
gesamten Beobachtungszeitraum. Die nach einheitlichen Factor Score Coefficients ermittelten Faktorenwerte ändern sich nur mehr wegen der tatsächlichen Variation der Ausgangsvariablen und sind daher absolut vergleichbar. In der vorliegenden Studie wird dieses Verfahren übernommen.

Hier dienen die Wiener Zählgebiete als räumliche Beobachtungsgrundlage, die kleinsten Einheiten (insgesamt 1359 ), für welche die verwendeten statistischen Daten vorliegen. Allerdings bedingen die Feinheit des räumlichen Bezugsrasters und die ungleichen GröBenverhältnisse seiner Elemente (manche Zählgebiete, besonders im dicht bebauten Gebiet, umfassen nur wenige Baublöcke, während an der Peripherie sehr viel größere Einheiten zusammengefasst sind) auch das häufigere Auftreten von "Einzelfällen“ mit besonderen, von der Umgebung stark abweichenden Strukturmerkmalen, oft auf Grund einer sehr individuellen Entwicklung. Um die „zufälligen“ Ausreißer möglichst zu unterdrücken und um die homogenen Zonen und ihre Übergänge deutlicher herauszufiltern, werden die Ergebnisse der historischen Faktorenanalysen hier als Modelle in Form von Trendflächen dargestellt. Damit soll eine modellhafte Verdeutlichung der Grundmuster der sozialräumlichen Strukturen und ihrer Veränderungen erreicht werden. Zur Berechnung der Trendflächen kam - unter Benutzung des Softwarepakets ArcView - die Methode der sog. Polygon-Filter (K. Neudecker, 1999) zur Anwendung: Hier sind Nachbarschaftsbeziehungen im räumlichen Muster der Zählgebiete über „Schablonen" definiert, die ein zentrales (Bezugs-)Polygon enthalten (das jeweils betrachtete Zählgebiet), welches von Nachbarpolygonen (= umliegende Zählgebiete) erster Ordnung (= unmittelbare Nachbarn) oder höherer Ordnung (= weitere „Nachbarschaftsringe") umgeben ist. Die Nachbarschaft wird über gemeinsame Grenzlinien (arc-adjacent) bzw. gemeinsame „Grenzecken“ (point-adjacent) definiert. Für die in einer solchen "Schablone" jeweils enthaltenen Zählgebiete lassen sich nun (arithmetische) Mittelwerte der verschiedenen, zu analysierenden Daten im vorliegenden Fall Faktorenwerte der sozioökonomischen, demographischen und ethnischen Struktur - errechnen. Sie beziehen sich jeweils auf das zentrale Polygon und stellen die Elemente der Trendflächen dar, in denen individuelle und "zufällige" Ausreißer unterdrückt und Übergänge "geglättet" werden. Somit ergeben sich generalisierte Modelle der Stadtstrukturen und ihrer Veränderungen.

\section{Historische Sozialraumanalyse}

\subsection{Grunddimensionen}

Nach der dargestellten Methode wurde auf der räumlichen Basis der Zählgebiete für das Wiener Stadtgebiet eine Faktorenanalyse über die Zeitreihe 1971-1981-1991 durchgeführt, also über drei Erhebungszeitpunkte und einen Beobachtungszeitraum von 20 Jahren. Die Auswahl der Inputdaten blieb beschränkt; sie mussten ja in allen drei Großzählungen des Beobachtungszeitraumes verfügbar sein. Dennoch sind hier aber alle Kennzahlen berücksichtigt, die sich in der relativ langen Tradition der Faktorialökologie immer wieder als wichtig erwiesen haben. Als Rechenverfahren für die historische Faktorenanalyse wurde die Standardversion mit VARIMAX-Rotation und KAISER-Normalisierung verwendet.

Tabelle 1 enthält die Matrix der Faktorenladungen und gibt somit Aufschluss über die statistischen Beziehungen zwischen den Inputvariablen und den ermittelten Faktoren:

- Der Faktor der sozioökonomischen Struktur wird vor allem gebildet durch hohe positive Ladungswerte der gehobenen Ausbildung sowie durch hohe negative Ladungen des niedrigsten Bildungsniveaus, gekoppelt mit entsprechenden Faktorladungen für gute bzw. schlechte Wohnverhältnisse. In räumlicher Dimension kennzeichnen hohe Faktorenwerte dieses komplexen Indikators also Wohnquartiere der sozialen Oberschichten, niedrige Werte die Wohnstandorte der Grundschichten.

- Die Dimension der demographischen Struktur ist bestimmt durch hohe positive Ladungswerte der Anteile von über 65-Jährigen, Einpersonenhaushalten und Frauen sowie durch hohe negative Ladungen der jüngeren Bevölkerung und der Mehrpersonenhaushalte. Somit weisen hohe Faktorenwerte für die demographische Struktur auf die Überalterung von Wohngebieten hin bzw. auf das häufigere Auftreten allein lebender Personen und Kleinfamilien, niedrige Faktorenwerte hingegen auf das Vorherrschen von jüngerer Bevölkerung und von kinderreicheren Haushalten.

- Als Leitvariablen der dritten sozialräumlichen Dimension - der ethnischen Struktur - erkennt man den Anteil ausländischer Wohnbevölkerung und - ebenfalls mit sehr hohen positiven Ladungswerten - die schlecht ausgestatteten Wohnungen. Hier dokumentieren sich die Lebensbedingungen der ausländischen Mitbürger, umso mehr, als auch noch der Anteil der Kleinwohnungen durch eine überdurchschnittlich positive Ladung gekenn- 
Tabelle 1 Grunddimensionen der historischen Faktorenanalyse (1971-1981-1991)

\begin{tabular}{|c|c|c|c|c|}
\hline \multicolumn{2}{|c|}{ Inputvariablen } & \multicolumn{3}{|c|}{ Faktoren (Variablenbündel) ${ }^{*}$} \\
\hline $\begin{array}{l}\text { Anteile } \\
\text { in } \% \text { an }\end{array}$ & Bezeichnung & $\begin{array}{l}\text { sozioökonomische } \\
\text { Struktur }\end{array}$ & $\begin{array}{c}\text { demographische } \\
\text { Struktur }\end{array}$ & $\begin{array}{l}\text { ethnische } \\
\text { Struktur }\end{array}$ \\
\hline \multirow{4}{*}{ Wohnbevölkerung } & Frauen & - & ++ & - \\
\hline & unter 15-Jährige & - & --- & - \\
\hline & über 65-Jährige & - & +++ & - \\
\hline & Ausländer & - & - & +++ \\
\hline \multirow{2}{*}{$\begin{array}{c}\text { Wohnbevölkerung } \\
\text { nach der höchsten } \\
\text { abgeschlossenen } \\
\text { Schulbildung }\end{array}$} & $\begin{array}{l}\text { Hochschule und } \\
\text { höhere Schule }\end{array}$ & +++ & - & - \\
\hline & Pflichtschule & --- & - & - \\
\hline \multirow{2}{*}{ Haushalte } & $\begin{array}{l}\text { 1-Personen- } \\
\text { haushalte }\end{array}$ & - & +++ & + \\
\hline & $\begin{array}{c}\text { 4- und -mehr- } \\
\text { Personenhaushalte }\end{array}$ & - & --- & - \\
\hline \multirow{4}{*}{ Wohnungen } & $\begin{array}{c}\text { Wohnungen mit } \\
\text { Wasser, Bad, WC, } \\
\text { Zentralheizung }\end{array}$ & ++ & - & - \\
\hline & $\begin{array}{l}\text { Wohnungen mit } \\
\text { und ohne } \\
\text { Wasseranschluss }\end{array}$ & - & - & +++ \\
\hline & $\begin{array}{l}\text { bis } 35 \mathrm{~m}^{2} \\
\text { Nutzfläche }\end{array}$ & -- & - & + \\
\hline & $\begin{array}{l}\text { über } 90 \mathrm{~m}^{2} \\
\text { Nutzfläche }\end{array}$ & +++ & - & - \\
\hline \multirow{2}{*}{$\begin{array}{c}\text { erklärte } \\
\text { Varianzanteile }\end{array}$} & je Faktor & 32,5 & 20,6 & 13,6 \\
\hline & kumulativ & 32,5 & 53,1 & 66,7 \\
\hline * Faktorenladungen: & $\begin{array}{l}+++: \text { über } 0,7 \\
-:-0,5 \text { bis }-0,3\end{array}$ & $\begin{array}{l}++: 0,5 \text { bis } 0,7 \\
--:-0,7 \text { bis }-0,5\end{array}$ & $\begin{array}{l}+: 0,3 \text { bis } 0,5 \\
---: \text { unter }-0,7\end{array}$ & 0,3 bis $-0,3$ \\
\hline
\end{tabular}

zeichnet ist. Positive Ladungen für die Einpersonenhaushalte und negative für den Frauenanteil weisen auf die demographischen Merkmale in den „Gastarbeiterquartieren“ hin, die durch höhere Faktorenwerte dieser Dimension hervortreten.

Die drei sozialräumlichen Dimensionen repräsentieren gemeinsam zwei Drittel der Varianz ihrer Ausgangsvariablen.

\subsection{Verbreitung der Grunddimensionen}

Ausmaß der Generalisierung

in den Trendflächenmodellen

Die Abbildungen 1 bis 6 zeigen die Ergebnisse der Trendflächenmodelle, dargestellt in der Form von „immateriellen Oberflächen“ als „,soziale“, „demographische" und "ethnische Gebirge", wobei auch die konventionellen Farben der Höhenstufen aus der topographischen Kartographie Verwendung finden. Über (Pearson-)Korrelationskoeffizienten zwischen der ursprünglichen Ausprägung der komplexen Indikatoren (Faktorenwerte) und den Modellvariablen kann das Ausmaß der Generalisierung in den Trendflächenmodellen erfasst werden. Es zeigt sich, dass bei der Dimension der sozioökonomischen Struktur die relativ größte Übereinstimmung zwischen der Ausgangssituation und dem Trendflächenmodell besteht $(r=0,84)$, d.h. die sehr ausgeprägten Grenzen zwischen den Wohnquartieren der verschiedenen sozialen Gruppen müssen im Trendflächenmodell nur relativ wenig generalisiert werden. Hingegen ergibt sich für die demographische Struktur eine deutlich größere Abweichung $(r=0,63)$ : Zurückzuführen ist dies auf den demographischen Wandel in der Kernstadt, der sehr differenziert verläuft, d.h. in benachbarten Zählgebieten, Baublöcken und sogar einzelnen Gebäuden vollzieht sich der zeitlich oft versetzte Austausch der Bevölkerung durch Sterbefälle, Abwanderung, Sanierung und Zuzug neuer Einwohner. Die entsprechenden Generalisierungen erfolgen allerdings nur so kleinräumig, dass neu entstandene, sehr komplexe demographische Strukturen nicht „verwischt" werden. Für die ethnische Struktur liegt der Korrelationskoeffizient $(r=0,73)$ zwischen den Werten der beiden anderen Dimensionen: Hier ist wohl 
besonders das partielle und "punkthafte" Eindringen der ausländischen Wohnbevölkerung in schlechter erhaltene Baugebiete und Gebäude, besonders im „inneren" Ring der Mittelschichtwohngebiete, für die Abweichung verantwortlich.

\section{Sozioökonomische Struktur}

In den Abbildungen 1 und 2 sind die Trendflächenmodelle der sozioökonomischen Struktur für 1991 und 1971 dargestellt, als „soziale Gebirge“ mit den höchstrangigen Wohnstandorten als „Gipfel“ und den Quartieren der Grundschicht als „Täler“. Das „Gipfelplateau" mit seiner (hypothetischen) "Gletscherregion“ erstreckt sich über die historische Altstadt und die Ringstraßenzone in den Cityrandbereich. In all diesen Bereichen kommen Gesetzmäßigkeiten der oberen Segmente des Wohnungsmarktes zum Tragen, vor allem die beschleunigten Erneuerungszyklen von Gebäuden und Wohnungen zum Abbau von „Rent Gaps“ ( M. Smith, 1987) zwischen exklusiven Ausstattungsmerkmalen der "Wohnumwelt" (bauliche Ensembles, Parks und Grünanlagen, soziale und demographische Struktur) und den angebotenen Wohneinheiten. Hier ist das Auftreten von sanierungsbedürftigen Gebäuden eher die Ausnahme und erklärt sich zumeist aus individuellen und besonderen Gegebenheiten. Der Abfall des „Gipfelplateaus“ reicht über die Zone der ehemaligen Vorstädte (die ihr sozialhistorisches Erbe als Mittelschicht- und z.T. auch Gewerbequartier bisher ebenfalls bewahrt haben, mit "Oberschichtkernen" um ehemalige Schlossund Parkanlagen, als Relikte der Peripherie der barocken Stadt) bis zum Straßenring des Gürtels. Hier kann man die scharfe Grenze zur Zone der ehemaligen "gründerzeitlichen“, d.h. aus der Epoche der Industrialisierung stammenden, Arbeiterwohnquartiere durch den abrupten Übergang in eine "soziale Ebene“ eindrucksvoll erkennen: Sie ist heute noch von Angehörigen der sozialen Grundschicht besetzt, darunter auch vom Großteil der ausländischen Mitbürger, die sich auf den schlechtesten Standorten konzentrieren. Am Außenrand dieses Ringes der Kleinwohnungen liegt die (ehemalige) Stadtrandindustrie, ein schmaler, von der Wohnbebauung „überfahrener" Streifen im Westen und eine breitere, an große Bahnanlagen gebundene Zone im Süden und Südosten, welche die Wohnquartiere z.T. vom übrigen Stadtgebiet abschottet. Diese Relikte der gründerzeitlichen Peripherie tragen dazu bei, dass die Lage- und Umwelteigenschaften der Standorte keinen Sanierungsanreiz bilden und Desinvestitionsstrategien der Anbieter auf dem Wohnungsmarkt begünstigen (bei zunehmender Nachfra- ge - besonders durch den Zuzug der ausländischen Bevölkerung - kann das Mietniveau sukzessive erhöht werden, auch bei fortschreitendem Verfall und trotz der unterlassenen Investitionen). Auch im Westen wo sich die Hänge des Wienerwaldes als attraktive Wohnlagen über die tertiären Flussterrassen (auf denen die Altstadt und die drei- bis fünfgeschossigen gründerzeitlichen Mietwohnhausgebiete liegen) erheben - ändert sich die sozioökonomische Struktur meist wieder auf sehr engem Raum. Gut zu erkennen ist die steile „Aufwölbung“ des sozialen Gebirges der Weststadt (Gliederung nach E. Lichtenberger; H. Fassmann; D. Mühlgassner, 1987) mit den älteren Kernen der gründerzeitlichen Villenperipherie im Anschluss an die alten Weinbaudörfer. Die Arbeiterwohnbebauung entlang des Wien-Flusses reicht keilförmig in die "Weststadt" hinein (siehe das tiefe „Tal“ in Abb. 1). Hingegen wird im Nordwesten der Ring des Arbeiterwohngebietes auf ein schmales Stück unterbrochen (als „Gebirgsrücken“ in Abb. 1), wo das Universitätsund Ärzteviertel (um das Allgemeine Krankenhaus), Folgenutzungen barocker Sommerresidenzen u.a. die Brücke vom inneren Cityerweiterungsgebiet zu den Villenvierteln schlagen. Die beiden jüngsten, äußeren Schalen der Stadtentwicklung - die Südstadt und die Oststadt - setzen sich als "Hügelländer“ von der „Ebene“ des Arbeiterwohnringes etwas ab. Im Detail sind sie sehr vielfältig gestaltet, als Mix aus gründerund zwischenkriegszeitlichen Quartieren mit mehrgeschossigen Mietwohnhäusern, mehr oder minder verstädterten Dörfern, größeren Trabantenstädten des sozialen Wohnbaus, errichtet in den 70er und 80er Jahren (z.T. als genossenschaftliche Wohnprojekte mit Mittelschichtbevölkerung, z.T. aber auch erst in jüngerer Vergangenheit) sowie schließlich aus der einsetzenden modernen, suburbanen Einfamilienhausperipherie.

Aus dem Vergleich der Trendflächenmodelle von 1991 (Abb. 1) und 1971 (Abb. 2) ergibt sich der Überblick über die Veränderungen der sozioökonomischen Struktur. Hier zeigen sich zunächst die Tendenzen zur generellen sozioökonomischen Aufwertung durch die allgemeine Erhöhung des Bildungsniveaus und die Verbesserung der beruflichen Qualifikationen („Fahrstuhleffekt"). Besonders betroffen sind die Weststadt, der östliche Rand der äußeren Entwicklungsschale und z.T. auch der südliche Teil des Stadtrandes. Auch am westlichen Cityrand und in den anschließenden Mittelschichtwohngebieten wirken sich die umfangreichen Sanierungs- und Parifizierungsmaßnahmen entsprechend aus, begünstigt durch die steigende „Urbanität“ (Theater, Trendgastronomie, revitalisierte Geschäftsstraßen usw.). Solche Prozesse haben 
Abbildung 1

„Soziales Gebirge“ 1991

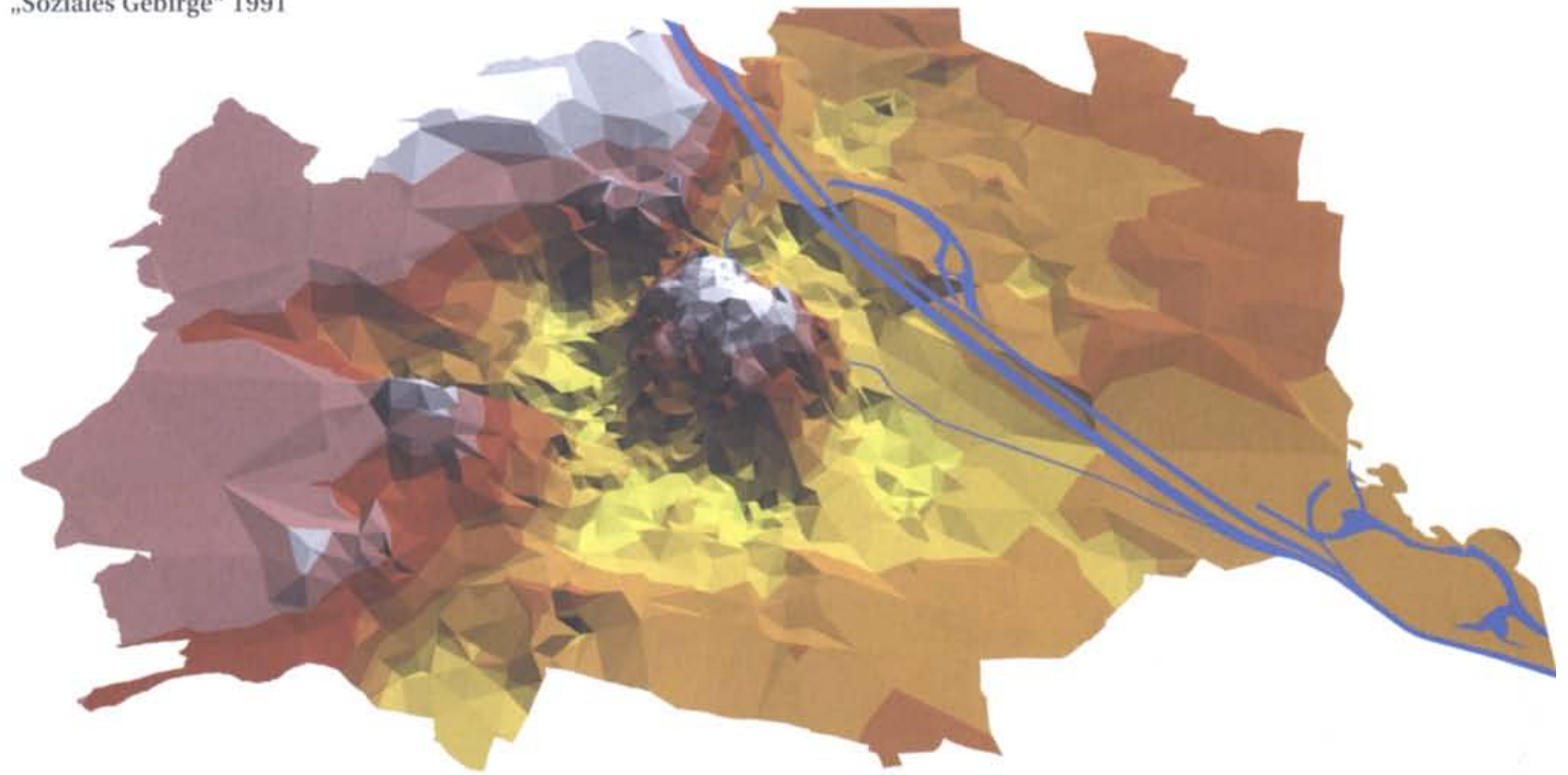

Sozioökonomische Struktur 1991 - Faktorenwerte

die Zahlen definieren jeweils den höchsten Wert der Klasse

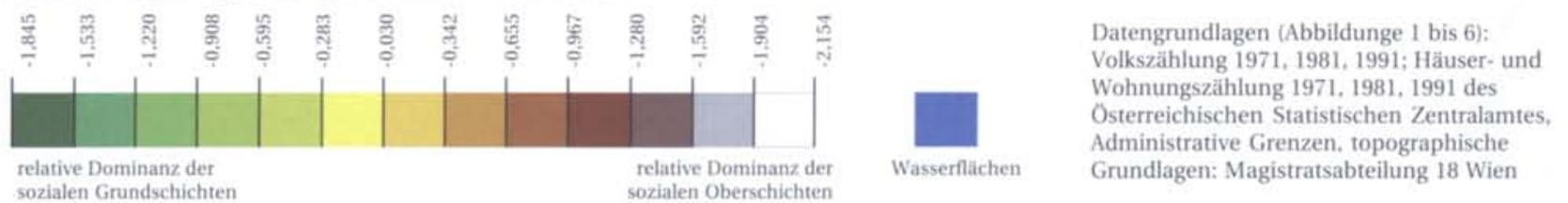

sozialen Grundschichten

Administrative Grenzen, topographische

Abbildung 2

"Soziales Gebirge" 1971

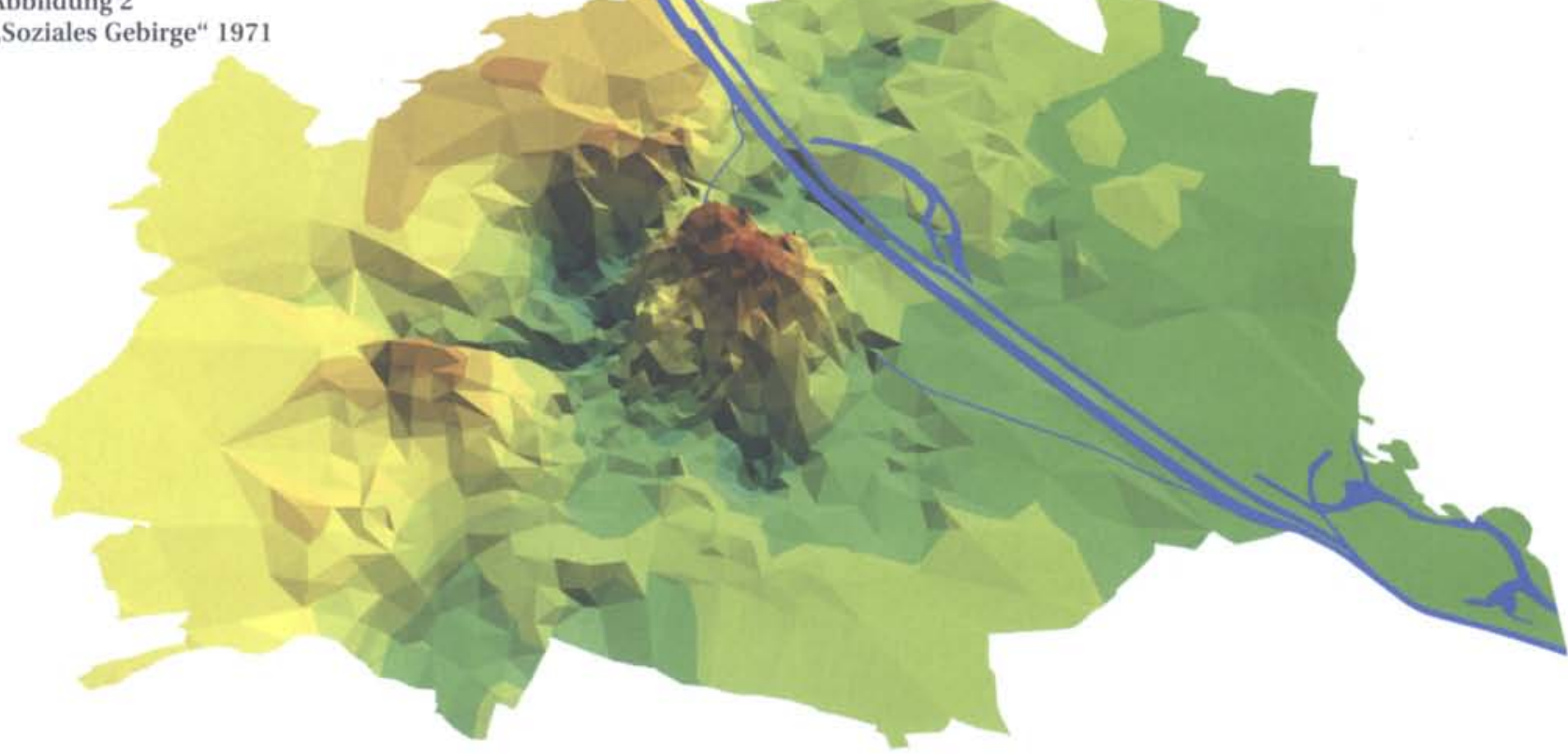

Sozioökonomische Struktur 1971 - Faktorenwerte

die Zahlen definieren jeweils den hôchsten Wert der Klasse

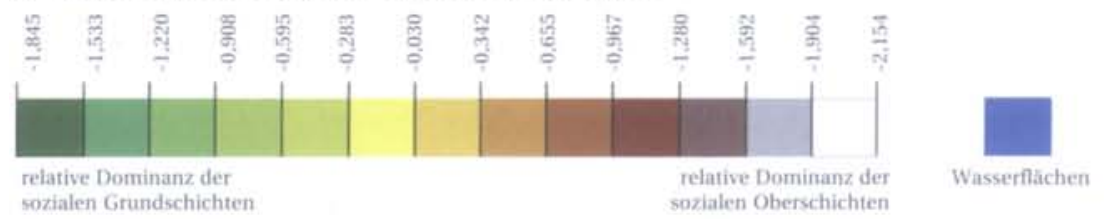


Abbildung 3

„Demographisches Gebirge" 1991

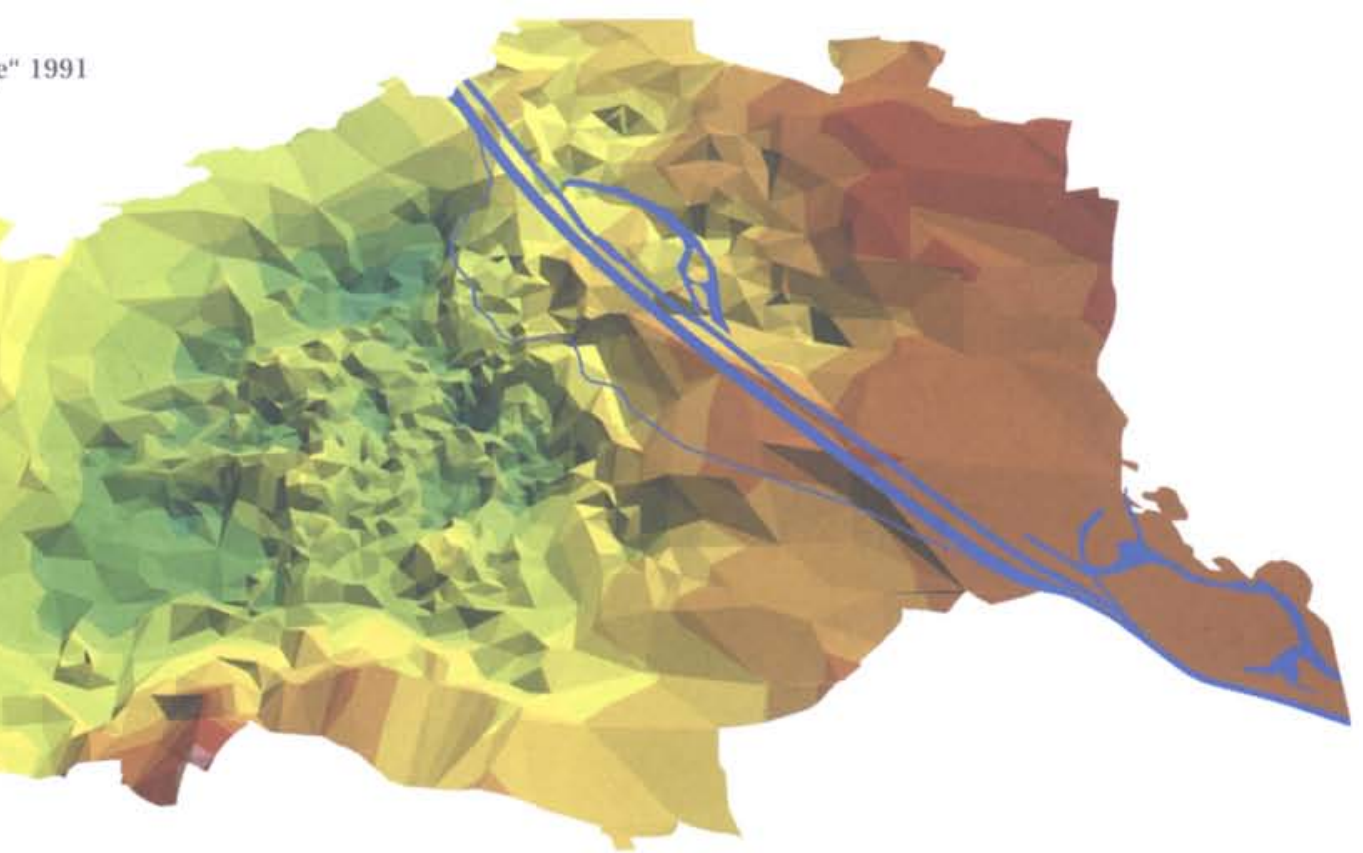

Demographische Struktur 1991 - Faktorenwerte

die Zahlen definieren jeweils den höchsten Wert der Klasse

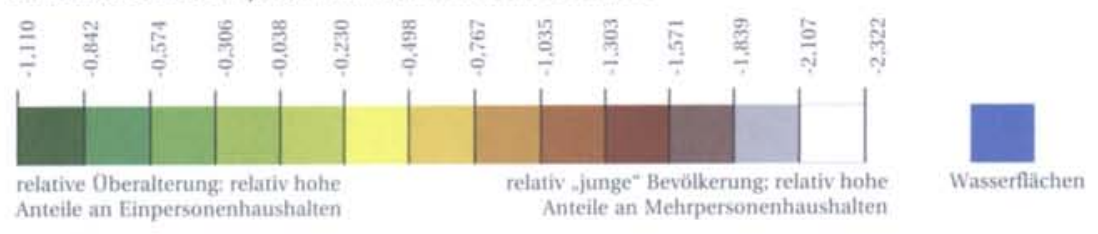

Abbildung 4

„Demographisches Gebirge" 1971

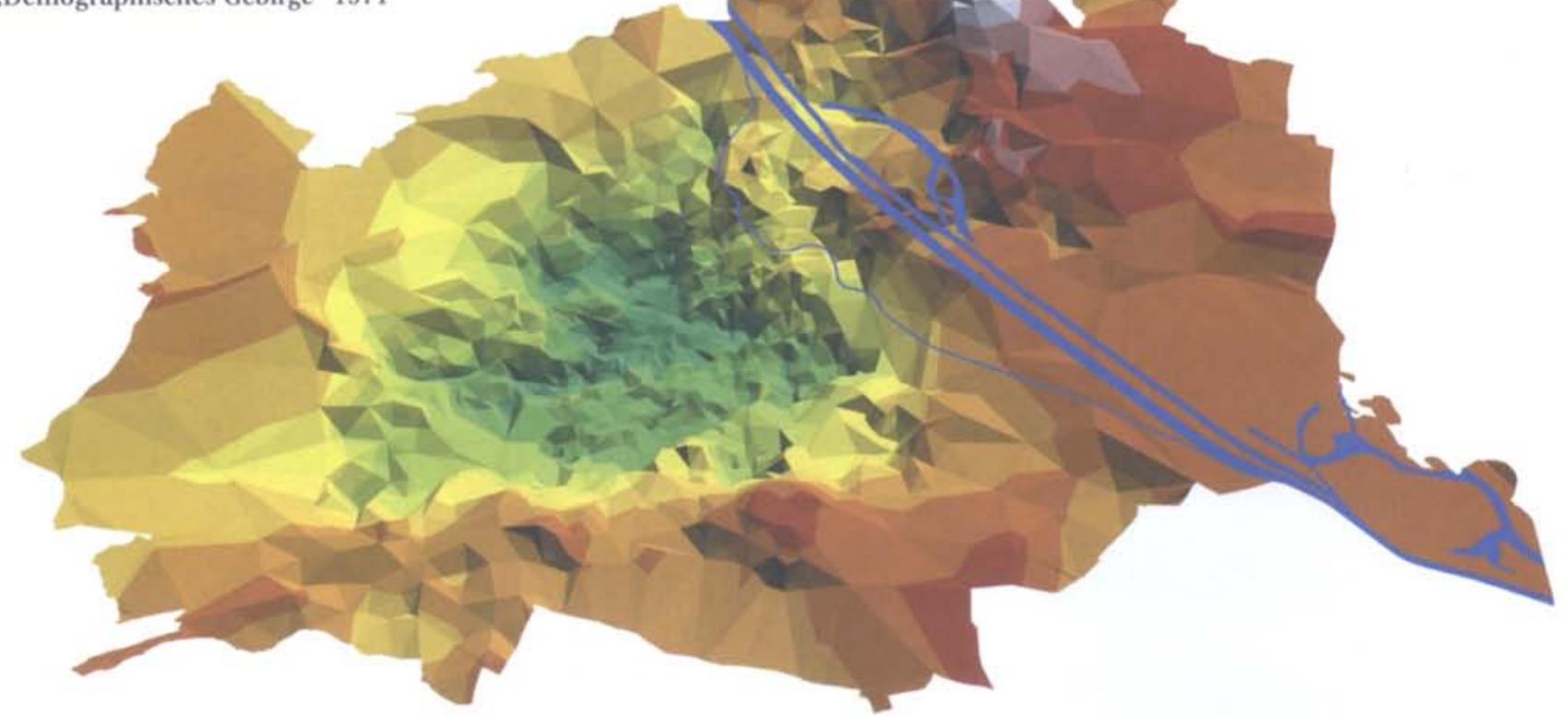

Demographische Struktur 1971 - Faktorenwerte

die Zahlen definieren jeweils den höchsten Wert der Klasse

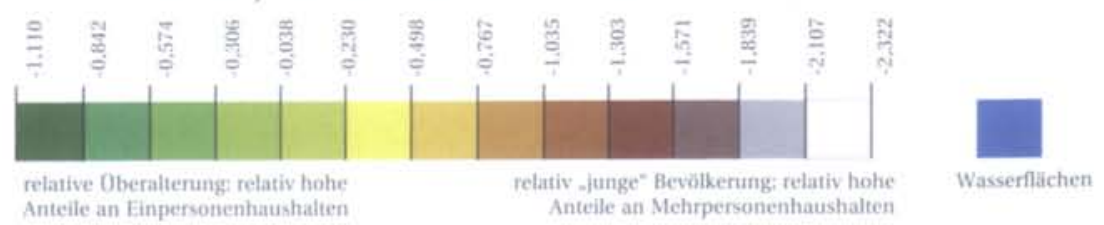


auch im Ring der Arbeiterwohngebiete eingesetzt, vor allem auf den nordwestlichen Standorten mit besseren Umweltbedingungen (Nähe zu attraktiven Parkund Grünanlagen, Nachbarschaft von Wohngebieten mit positivem Image, neue Lokalszene u.a.) als Voraussetzung für den kommerziellen Erfolg der Investitionen (Aufstieg in das mittlere Segment des Wohnungsmarktes). Die „Hauptkerne“ des Arbeiterwohngebietes bleiben aber deutlich hinter dem allgemeinen Aufwärtstrend zurück. Hier wirken sich die Desinvestitionsstrategien der Anbieter im untersten Segment des Wohnungsmarktes aus.

\section{Demographische Struktur}

Im Trendflächenmodell der demographischen Struktur für 1991 (Abb. 3) bildet das „demographische Gebirge" die jüngere, kinderreichere Bevölkerung (größere Haushalte, geringer Anteil berufstätiger Frauen) ab, während die „Ebenen“ Wohnquartiere mit Überalterungsmerkmalen (plus erhöhten Anteilen von Einfamilienhaushalten und Frauen) kennzeichnen. Es zeigen sich gewisse Übereinstimmungen mit dem Modell der amerikanischen Sozialraumanalyse (relativ ,junge“ demographische Strukturen an der Peripherie, Überalterungsphänomene im Kern). Allerdings wird dieses Bild, wie auch in anderen europäischen Metropolen, durch historische Zyklen des demographischen Wandels gestört: Eine wesentliche Abweichung ergibt sich durch die Dominanz älterer Bevölkerungsgruppen in der peripheren Oberschichtzone der Weststadt (als "Ebene“ in Abb. 3). Auch das Wiental als Arbeiterwohngebiet, welches - wie oben gezeigt - „keilförmig“ in die Oberschichtzone hineinragt, unterscheidet sich hier nicht von seiner Umgebung: Die benachbarten Ober- und Grundschichtquartiere sind gleichermaßen überaltert. Die zweite Abweichung vom demographischen Modell der traditionellen Sozialraumanalyse stellt das Aufbrechen der Überalterung im Kernbereich dar, wobei diese z.T. mit dem sozialen Wandel in den Sanierungsgebieten zusammenfällt, z.T. aber auch durch das Eindringen von jüngerer ausländischer Bevölkerung in die noch nicht erneuerten Quartiere des Arbeiterwohngebietes bedingt ist. Im „demographischen Gebirge" der äußeren Schale der Stadtentwicklung sind die großen Trabantenstädte und Wohnsiedlungen der Südstadt Anfang der 90er Jahre schon in ihr demographisches Reifestadium eingetreten (nur für die jüngeren Anlagen des sozialen Wohnbaus im Südosten trifft das noch nicht zu), während die Oststadt im Alterungszyklus noch etwas zurückliegt.
Abbildung 4 enthält das demographische Trendflächenmodell für 1971. Der Vergleich mit Abbildung 3 zeigt recht eindrucksvoll den beginnenden Aufbau eines neuen „demographischen Gebirges“ („Hügellandes“) im Arbeiter- und z.T. auch im Mittelschichtring. Hier sterben die relativ gleichaltrigen Bewohner innerhalb eines kürzeren Zeitraumes aus, die wegen der regulierten Mieten und den Mieterschutzbestimmungen oft einen großen Teil ihres Lebens in den Gebäuden verbracht haben (nur eher seltener werden sie aus ihren Wohnungen verdrängt). Im Sanierungsfall kommt es zu gekoppelten „Verjüngungszyklen" der Bevölkerung und der Bausubstanz, allerdings weniger in den schlechten Wohnlagen: Hier müssen die nachziehenden jüngeren Bevölkerungsgruppen (oft arme Angehörige ethnischer oder nationaler Minderheiten, siehe oben) mit Wohnungen vorlieb nehmen, welche ihr „Filtering Down“ noch nicht beendet haben. In der Ost- und Südstadt sind die durch die „Alterungszyklen“ der Bevölkerung bedingten Strukturveränderungen zu erkennen.

\section{Ethnische Struktur}

In den Arbeiten der Sozialraumanalyse wird zumeist ein eher „punktuelles" räumliches Verbreitungsmuster der Dimension des "Segregationsgrades“ nachgewiesen. Vergleiche der Abbildungen 5 und 6, welche die ,ethnischen Reliefs" für 1991 und 1971 enthalten, zeigen, dass dies am Beginn der 70er Jahre in etwa noch der Fall war. Hier existieren solche „ethnischen Kerne“ ("Erhebungen“ in Abb. 6) in der Schale des gründerzeitlichen Arbeiterwohngebietes. An Hand des Trendflächenmodells für 1991 ist zu erkennen, dass sich in der zwanzigjährigen Beobachtungsperiode ausgehend von diesen „ethnischen Kernen“ eine weitgehend zusammenhängende „ethnische Zone“ (= „ethnisches Gebirge“ in Abb. 5) gebildet hat, welche sich über weite Teile des Kleinwohnungsringes erstreckt. Ein Vergleich mit der demographischen Entwicklung (Abb. 3) macht deutlich, dass die hier ablaufenden Verjüngungsprozesse (mit Ausnahme der oben dargestellten "Sanierungskerne" im Nordwesten) auf die zugewanderten jüngeren und kinderreicheren Ausländerfamilien zurückgehen. 
Abbildung 5

„Ethnisches Gebirge“ 1991

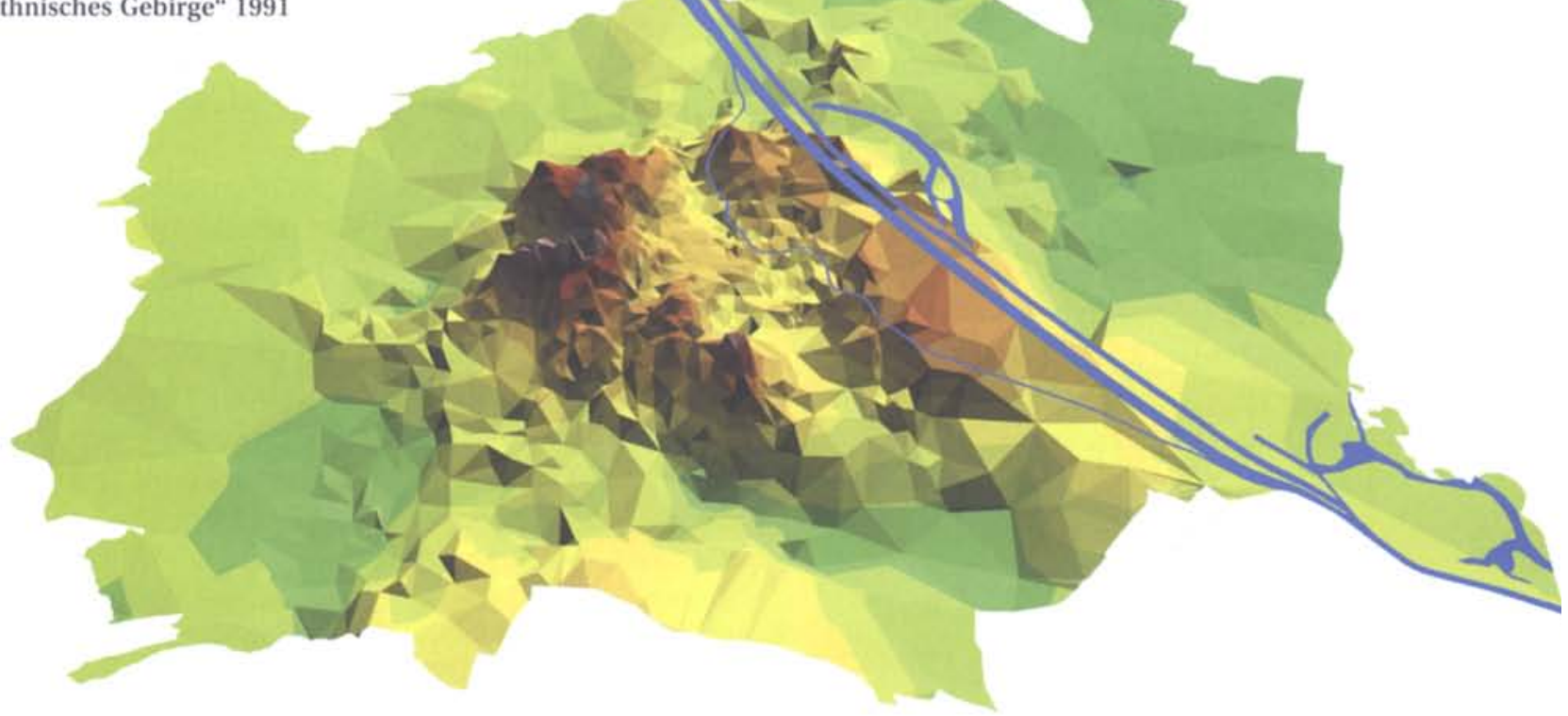

Ethnische Struktur 1991 - Faktorenwerte

die Zahlen definieren jeweils den höchsten Wert der Klasse

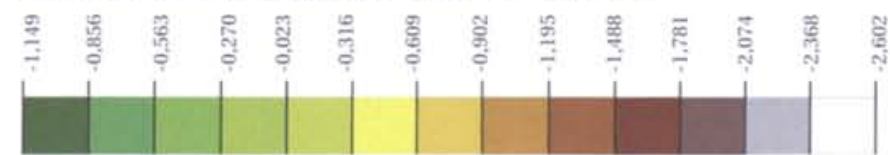

relativ nieder

Abbildung 6

„Ethnisches Gebirge“ 1971

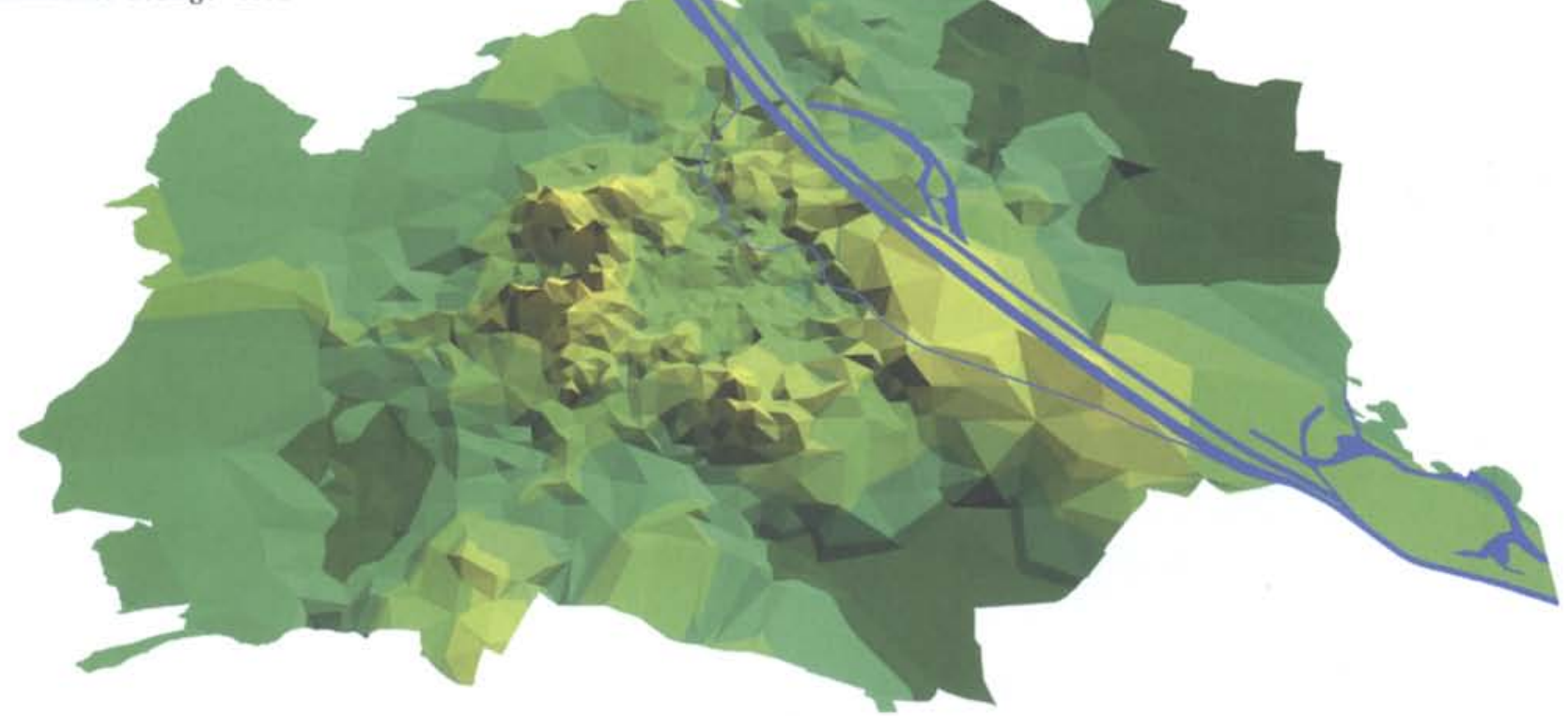

Ethnische Struktur 1971 - Faktorenwerte

die Zahlen definieren jeweils den höchsten Wert der Klasse

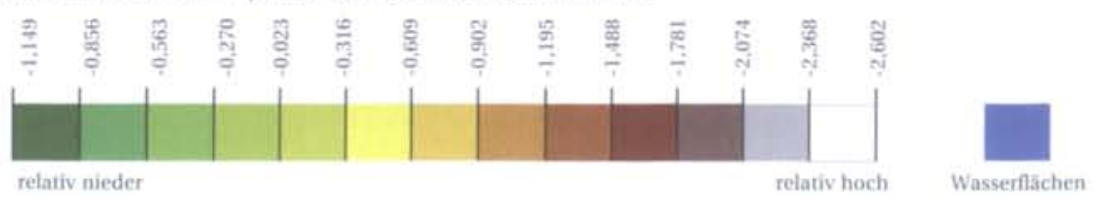




\section{Problemlagen}

Wie die Ergebnisse der historischen Sozialraumanalyse und der Trendflächenmodelle zeigen, stellen zwei Segregationsprozesse sowie die Auswirkungen der Alterungszyklen bedeutende Problemlagen der Wiener Stadtentwicklungsplanung dar.

Die fortschreitende Ghettoisierung der ausländischen Mitbürger - als der erste Segregationsprozess - resultiert vor allem aus:

- den in aller Regel zu hohen Einstiegskosten zur Inanspruchnahme auch der billigsten Angebote auf dem Markt der Eigentums- und Genossenschaftswohnungen;

- dem Ausschluss vom Sektor der Sozialwohnungen (Voraussetzung für die Bewerbung: österreichische Staatsbürgerschaft);

- der Abdrängung der Ausländer und ethnischen Gruppen in die privaten Mietwohnungsbestände der gründerzeitlichen Problemgebiete, wobei sich der für diese Nachfragergruppen verfügbare Wohnungsbestand wegen der oben dargestellten Sanierungsaktivitäten in den standörtlich attraktiveren Randbereichen sukzessive verringert. Dies fördert nicht nur die weitere Konzentration in den „Kernen" der Problemgebiete, sondern erhöht auch noch zusätzlich (siehe oben) das Mietniveau.

In einer Studie der Stadt + Regionalforschung GmbH über die Lebenssituation von Ausländerinnen in Wien (H. Feigelfeld, R. Hartig, 1997) zeigt es sich, dass $83 \%$ der untersuchten Haushalte in Althäusern wohnen, die vor 1945 errichtet wurden. Drei Viertel der Ausländerinnen leben unter der Mindestnorm an Wohnfläche (dagegen nur rund $20 \%$ der vergleichsweise untersuchten Inländerinnen) und $52 \%$ in schlecht ausgestatteten Substandardwohnungen. Nach einer Untersuchung des Wiener Instituts für Stadt- und Regionalforschung (1996) lag die durchschnittliche Quadratmetermiete von Substandardwohnungen für ausländische Mieter höher als die Miete einer Kategorie A-Wohnung für Österreicher!

Die räumliche Konzentration der ausländischen Mitbürger führt dazu, dass in den entsprechenden Wohnquartieren die fremde Kultur immer „sichtbarer" wird und das soziale Milieu zusehends unter die Kontrolle der Fremden gerät. Ernsthaftere soziale Konflikte entstehen mit denjenigen einheimischen Bevölkerungsgruppen, die auf Grund ihrer sozialen Schwäche dazu gezwungen sind, in den gründerzeitlichen Problemgebieten zu verbleiben, und sich zunehmend als Minderheit empfinden (nach K. Czasny, G. Bständig und R. Hartig, 1998, hat derzeit etwa jeder vierte inländische Wohnungswechsler keine andere Chance). Andererseits bestärken die hohen Kosten für abgewohnte und oft überbelegte Quartiere auch die Feindseligkeit der ausländischen Zuwanderer. Zusätzlich zu den wenig attraktiven Gestaltungsmerkmalen der baulichen Wohnumwelt entsteht so ein soziales Klima, das private Sanierungsinvestitionen wesentlich behindert. Es kommt zur "Zementierung" und Selbstverstärkung der Strukturen. Auch wenn der nach der jüngsten Bevölkerungsprognose (J. Kytir, G. Lebhart, A. Kaufmann, A. Hanika, J. Urlich, 1998) wegen der restriktiven Zuwanderungspolitik - zu erwartende leichte Rückgang der ausländischen Bevölkerung (Ende 1999: ca. 285 000; 2021: ca. 262 000) tatsächlich zutreffen sollte (was aus verschiedenen Gründen bezweifelt werden kann, siehe etwa J. Steinbach, A. Holzhauser, K. Neudecker, 2000), werden die fremden Mitbewohner (plus ihre nach den Prognoseannahmen bereits eingebürgerten, aber wohl noch nicht zur Gänze integrierten ehemaligen Landsleute) ein "Problempotenzial” bilden, das ohne flankierende politische Maßnahmen sehr relevant werden dürfte. Somit bestehen zukünftig mit größerer Wahrscheinlichkeit wohl nur die beiden stadtentwicklungspolitischen Alternativen:

(1)

Die Fortsetzung des gegenwärtigen Trends der Konzentration und Segregation in den Problemgebieten, wo sich bei nur unzureichend gebremstem baulichen Verfall die Dominanz der fremden Kulturen immer mehr erhöht. Schon zwischen 1991 (dem letzten Bezugsjahr der Sozialraumanalyse) und dem Anfang des Jahres 2000 haben sich die Anteile der ausländischen Bevölkerung in denjenigen Wiener Stadtbezirken, wo sich die gründerzeitlichen Problemgebiete konzentrieren (15. Rudolfheim-Fünfhaus, 16. Ottakring, 17. Hernals und 20. Brigittenau), um 7 bis 9,5 Prozentpunkte erhöht und liegen nun zwischen 26 und $33 \%$ (Angaben für die viel kleineren Einheiten der Zählgebiete mit z.T. wesentlich höheren Anteilswerten sind erst nach den Großzählungen von 2001 erhältlich). Diese Konzentrationsprozesse fördern die fortschreitende Reduktion der sozialen Beziehungen zwischen einheimischen und ausländischen Bevölkerungsgruppen auf die notwendigen „Vollzugsnormen" (meist berufliche, hierarchische und routinisierte Kontakte zur Aufrechterhaltung wirtschaftlicher Funktionen). Es kommt zur Ausbildung von restringierten städtischen Interaktionsmustern, wobei die Mehrheitsbevölkerung schließlich die fremden „Enklaven” meidet, während die privaten Aktionsräu- 
me der Minderheit weitgehend auf ihr Wohnumfeld beschränkt bleiben (J. Steinbach, 1998). Gerade dieser verstärkte Ethnozentrismus fördert aber wieder die Gefahr von sozialen und politischen Konflikten, besonders an den „ethnischen” Grenzen, der dann unter Umständen durch Strategien der sog. "colonization of space" (J. Short, 1996) begegnet wird: z.B. intensive Kontrolle und Bewachung durch die Polizei und private Wachdienste oder städtebauliche Konzepte („bunker architecture”) zur Abriegelung der Problemgebiete. Somit werden die räumlichen und sozialen Disparitäten konserviert und vermindern langfristig die Lebensqualität der Bevölkerung und die wirtschaftliche Standortqualität der Stadt.

\section{(2)}

Maßnahmen der Stadtentwicklungspolitik, die auf die sukzessive Verminderung der Segregation in den gründerzeitlichen Problemgebieten abzielen, vor allem durch die soziale, politische (Einbürgerung) und kulturelle Integration (z.B. Eröffnung von Chancen zur qualifizierteren Bildung) eines Teiles der ausländischen Mitbürger, welche ihnen auch den Wohnungswechsel in andere Stadtgebiete (d.h. die Partizipation an zusätzlichen Sektoren des Wohnungsmarktes) erleichtert. Natürlich reichen dazu Maßnahmen der Stadtentwicklungspolitik allein nicht aus: Sie müssen durch ein ganzes Spektrum komplementärer Politiken ergänzt werden (von der Einwanderungs- und Arbeitsmarktpolitik über die Wohnungs- und Sozialpolitik bis in die Bereiche von Kultur- und Unterrichtspolitik). Im Fall der Realisierung solcher Konzepte von ,integrierten Integrationspolitiken" bliebe zwar die Funktion der Kleinwohnungsgebiete als Stätten der kulturellen Transformation vermutlich erhalten. Sie könnten aber viel mehr als bisher in die jüngeren Sanierungsund Umbauzyklen mit einbezogen werden und sich vielleicht $\mathrm{zu}$ attraktiveren Wohn- und Dienstleistungsstandorten mit „multiethnischem Flair” entwickeln.

Allerdings setzt die Realisierung der zweiten Alternative zunächst die Änderung des politischen Klimas und den Abbau von ethnozentrischen Vorurteilen voraus. Angesichts der ablaufenden massiven Veränderungen von wirtschaftlichen und gesellschaftlichen Rahmenbedingungen dürften aber dazu derzeit keine allzu großen Chancen bestehen. Eine Entwicklung in Richtung der skizzierten ersten Variante erscheint als eher wahrscheinlich.

Der zweite Segregationsprozess im Wiener Stadtgebiet, welcher sich anhand der Ergebnisse der historischen Faktorenanalyse und der Trendflächenmodelle erken- nen lässt, bezieht sich auf die sozial schwächeren inländischen Bevölkerungsgruppen, denen ebenfalls der Zugang zu den meisten Segmenten des Wohnungsmarktes verwehrt bleibt. Ihre Partizipationschancen werden von K. Czasny (1999) folgendermaßen beurteilt: Etwa 40 \% der Wohnungswechsler sind wegen zu geringer Ersparnisse vom gesamten Neubauangebot ausgeschlossen (und daher auf Sozialwohnungen bzw. auf geförderte und nicht geförderte ältere Mietwohnungen angewiesen), etwas mehr als ein Drittel der Nachfrager gehört zum Zielpublikum der mit öffentlicher Wohnbauförderung errichteten Neubauwohnungen und nur das restliche Viertel kann auch am frei finanzierten Wohnbausektor partizipieren. Daher konzentrieren sich die sozial schwachen inländischen Haushalte zusehends:

- in den billigen, nicht sanierten, privaten Altmiethäusern mit wenig attraktivem Wohnumfeld, oft in Nachbarschaft zu den ausländischen Mitbürgern sowie

- in den älteren Anlagen des sozialen Wohnbaus, da mittlerweile auch für die neueren Wohnungen der Stadt Wien das Mietniveau so angehoben wurde, dass vielen ärmeren Haushalten der Zugang verwehrt bleibt. So betragen nach K. Czasny (1999) die Anteile der Angehörigen unterer sozialer Schichten an den Wohnungen mit „kommunalen” Mieten, differenziert nach Baualtersklassen: vor 1919: $67 \%$; 1919-1945: $61 \%$; 1946-1968: $51 \%$; seit 1968: $39 \%$.

Somit drohen die älteren Sozialwohnungsbestände und die unattraktiven privaten Altwohnungen immer mehr zum „Auffanglager” für die sozial Schwächeren zu werden, so es nicht gelingt, durch Maßnahmen der Wohnungs- und Sozialpolitik gegenzusteuern.

Im Rahmen der Sozialraumanalysen konnte gezeigt werden, dass in der Mehrzahl der Wiener Wohnquartiere mehr oder minder deutlich ausgeprägte „Verjüngungstendenzen“ vorherrschen, was auch für den Stadtdurchschnitt zutrifft. Allerdings geht die jüngste Bevölkerungsprognose (J. Kytir, G. Lebhart, A. Kaufmann, A. Hanika, R. Ulrich, 1998) davon aus, dass ein neuer intensiver Veralterungszyklus wieder bevorsteht, wegen:

- der geringen Fertilität der inländischen Frauen in den letzten drei Jahrzehnten;

- der Alterung von geburtsstärkeren demographischen Kohorten: Die etwas stärker besetzten Jahrgänge von 1938 bis 1944 rücken schon derzeit in die Altersgruppe der über 60-Jährigen auf, und auch die „Baby-Boom-Generation” wird in zwanzig Jahren an der Schwelle zum Pensionsalter stehen;

- der steigenden Lebenserwartung. 
Wien soll im Jahr 2021 mit etwa 445000 über 60-Jährigen wieder annähernd gleich viele ältere Bewohner haben, wie dies schon Anfang der 70er Jahre der Fall war, und diese Anzahl wird voraussichtlich noch weiter steigen. Aber auch die Altersgruppe der 45- bis 60Jährigen dürfte deutlich zunehmen, während die Zahl der 30 - bis 45 -Jährigen stark zurückgeht und sich bei den jüngeren Gruppen vergleichsweise nur geringe Verschiebungen ergeben. Räumlich gesehen gleichen sich die Unterschiede in der Altersstruktur an, wobei auch die gründerzeitlichen Problemgebiete (heute: "demographisches Hügelland") wieder in den Überalterungsstatus der 70er Jahre zurückfallen sollen. Sowohl hier als auch in der Wachstumsschale der Südund Oststadt werden sich die Überalterung und die „doppelte Segregation“ gemeinsam negativ auswirken.

Denn auch die heute "neueren Wohnungsanlagen" in der Süd- und in der Oststadt sind zukünftig von den Überalterungsproblemen besonders betroffen. Hier wird sich ohne wesentliche planerische Eingriffe ein ganzes Spektrum von Strukturmerkmalen sehr ungünstig auswirken. Es reicht von der rudimentären altersmedizinischen Versorgung, der oft unzureichenden Anbindung im öffentlichen Nahverkehr sowie der vielfach unzulänglichen Nahversorgung bis hin zu den baulichen und sozialen Defiziten der nach den Konzepten der architektonischen Moderne errichteten Satellitenstädte. Trotz der freistehenden Bauweise der meist vielgeschossigen Wohngebäude fehlen hier oft Balkone, Loggien, Terrassen oder Mietergärten ebenso wie auf Kommunikation und Freizeit bezogene Einrichtungen. Diese wären hier sehr viel wichtiger als in den zentraleren Stadtbereichen, wegen des dünnen Besatzes mit Gaststätten, Unterhaltungs- bzw. Sozialeinrichtungen im Wohnumfeld und der meist großen Entfernung zu den wenigen städtischen Subzentren oder gar zur Innenstadt. Angesichts der bereits heute in diesen Großwohnanlagen festgestellten Kommunikationsdefizite und der mangelnden sozialen Integration (etwa bezüglich der für das Alltagsleben so wichtigen Beziehungen von mittlerer Distanz), der Bedenken bezüglich der persönlichen Sicherheit und der subjektiven Feindbilder (H. Feigelfeld, 1994) kann man sich die zukünftige Lebenssituation der alten (vermutlich weitgehend auch armen) Bevölkerung gut vorstellen.

Aber nicht nur für die gründerzeitlichen Problemgebiete und für die Trabantenstädte der Süd- und Oststadt bringt eine Verfestigung der Überalterung bis in die zweite Hälfte des 21. Jahrhunderts beträchtliche Probleme, sondern es ist auch für die Gesamtstadt ein ganzes Spektrum von negativen Konsequenzen zu erwarten. Dazu zählen nicht nur die drohende „Pensionslücke“, sondern vor allem auch der teilweise Ausfall des Potenzials an jüngeren und kreativen Trägern eines weiteren wirtschaftlichen Aufschwungs. Unter solchen Umständen erscheinen die radikalen Einwanderungsbeschränkungen - besonders aus den osteuropäischen Beitrittsländern der EU - als nur schwer verständlich. Es ist abzusehen, dass die westeuropäischen Metropolen schon in näherer Zukunft um den Zuzug von jüngeren und dynamischen Bevölkerungsgruppen konkurrieren werden, wobei vermutlich die nationale und ethnische Herkunft keine allzu große Rolle mehr spielt.

\section{Schlussbemerkung}

Mit den Methoden der historischen Sozialraumanalyse wurde im Rahmen der vorliegenden Arbeit versucht, Strukturmerkmale und Entwicklungstendenzen im Wiener Stadtgebiet aufzuzeigen und daraus resultierende stadtentwicklungspolitische Probleme zu identifizieren. Von ihrer Lösung hängt nicht nur die zukünf-tige Lebensqualität der Wiener Bevölkerung ganz wesentlich ab, sondern es werden dadurch auch wirtschaftliche Standortbedingungen gefördert, die im Rahmen der fortschreitenden Integration Westeuropas und der bevorstehenden Erweiterung des Wirtschaftsraumes der Europäischen Union durch die Reformstaaten Osteuropas eine immer größere Rolle spielen.

Unter diesem Aspekt scheint die Fortsetzung des dargestellten Forschungsansatzes wichtig, vor allem nach dem Vorliegen der Ergebnisse der nächsten amtlichen Großzählungen im Jahr 2001. Erst mit diesen Daten sind wieder kleinräumige Vergleiche möglich, und die historischen Sozialraumanalysen können über einen Zeitraum von dreißig Jahren durchgeführt werden. Dann sollte man auch das räumliche Bezugsgebiet über die Wiener Stadtgrenzen hinaus erweitern und den gesamten sozialen und wirtschaftlichen Funktionsraum der Bundeshauptstadt berücksichtigen. 


\section{Literatur}

Berry, B. J. L. (1972): City Classification Handbook. Methods and Application. London

Cserjan, K.; Sauberer, M. (1972): Sozialräumliche Gliederung Wien 1961. Ergebnisse einer Faktorenanalyse. In: Der Aufbau, 27. Jg., 7/8

Czasny, K. (1999): Soziale Struktureffekte der Wohnbauförderung. In: StadtRaumZeit 1

Czasny, K.; Bständig, G.; Hartig, R. (1998): Wohnungswechsel und Wohnungsnachfrage in Wien. In: StadtRaumZeit 1

Feigelfeld, H.; Hartig, R. (1997): Lebenssituation und Zufriedenheit von Ausländerinnen in Wien. In: StadtRaumZeit 2

Hatz, G. (1998): Sozialraumstrukturen und sozialer Wandel in Wien 1971-1991. In: Geographischer Jahresbericht aus Oster. reich, Bd. LV

Horvath, R. J. (1994): National Development Path 1965-1987: Measuring a Metaphor. In: Environment and Planning A, Vol. 26, No. 3

Institut für Stadt- und Regionalforschung (1996): Sozialdynamik und Wohnungsmilieu. Wien

Kytir, J.; Lebhart, G.; Kaufmann, A.; Hanika, A.; Ulrich, R. (1998): Bevölkerungsvorausschätzung 1996 bis 2021 nach Teilgebieten der Wiener Stadtregion. Werkstattberichte 23 (Stadtplanung Wien)

Lichtenberger, E. (1990): Stadtverfall und Stadterneuerung. Beiträge zur Stadt- und Regionalforschung, Bd. 10, Wien

Lichtenberger, E.; Fassmann, H.; Mühlgassner, D. (1987): Stadtentwicklung und dynamische Faktorialökologie. Beiträge zur Stadt- und Regionalforschung 8, Wien

Neudecker, $K$. (1999): Dreidimensionale Darstellung von natürlichen und immateriellen Oberflächen. Projektarbeit im Rahmen des UNIGIS ‘97-Lehrganges, Eichstätt, Salzburg
Österreichisches Statistisches Zentralamt, Ergebnisse der Volkszählungen sowie der Häuser- und Wohnungszählungen 1971, 1981, 1991 für Wien

Shevky, E.; Bell, W. (1955): Social Area Analysis: Theory, Illustrative Applications and Computational Procedures. New York

Short, $J$. (1996): The Urban Order. An Introduction to Cities, Culture and Power. Cambridge, MA.

Smith, $N$. (1987): Gentrification and the rent gap. In: Annals of the Association of American Geographers, 77

Statistisches Amt der Stadt Wien (1999): Wien - Statistik aktuell. Wien online

Steinbach, J.; Holzhauser, A.; Neudecker, K. (2000): Historische Sozialraumanalyse für das Wiener Stadtgebiet. Werkstattberichte 35 (Stadtplanung Wien)

Steinbach, J. (1998): Politische, ökonomische und räumliche Restriktionen im Verhalten von Minderheiten. In: Arbeiten aus dem Institut für Geographie der Karl-Franzens Universität Graz, Bd. 36

Steinbach, J.; Hilger, S. (1997): Erneuerungsstrategien für ausgewählte Geschäftszentren im dicht bebauten Wiener Stadtgebiet, Werkstattberichte 20 (Stadtplanung Wien). Stadtprofile 16a (Wirtschaftskammer Wien)

Überla, K. (1977): Faktorenanalyse. Berlin, Heidelberg, New York

Prof. Dr. Josef Steinbach

Dipl.-Geogr. Andrea Holzhauser

Dipl.-Geogr. Klaus Neudecker

Katholische Universität Eichstätt

Professur für Wirtschaftsgeographie

Ostenstraße 18

85072 Eichstätt

E-Mail: wirtschaftsgeographie@ku-eichstaett.de 